\title{
ATLAS Silicon Microstrip Tracker Operation
}

\author{
K. Hanagaki* \\ for the ATLAS SCT group \\ Graduate School of Science, Osaka University, Machikaneyama 1-1, Toyonaka, \\ Osaka 560-0043, Japan
}

\begin{abstract}
The Semi-Conductor Tracker (SCT) has been built and installed into the ATLAS detector at the Large Hadron Collider (LHC) in CERN. Together with the Pixel and Transition Radiation Tube (TRT) detectors it will provide precise tracking and vertexing information for the ATLAS Physics program. In this paper we report on the status, operational experience, and performance of the SCT in the run up to the start of the LHC.
\end{abstract}

Key words: Silicon Microstrip Tracker, ATLAS, Radiation damage, Noise PACS: 29.40.Gx, 29.40.Wk

\section{Introduction}

There are three charged particle tracking detectors in the ATLAS detector, as shown in Fig. 1. The innermost detector is the pixel detector for precise vertexing. The SCT is located outside of the pixel detector and is mainly for tracking and to improve the primary and secondary vertex finding. The outermost part is TRT for continuous tracking to large radius.

The SCT consists of a central barrel and two endcaps. The barrel and endcaps were installed into ATLAS cryostat in 2006, and 2007 respectively. The installation was followed by a period of commissioning including: the cooling, electrical and optical connectivity tests, calibrations (optical and electrical), Detector Control System and other system check out tests. After that, cosmic rays were used to tune the operation of the detector to be ready for the delivery of the first beam by the LHC.

* Corresponding author. ph +81-6-6850-5357
Email address: kazu@champ.hep.sci.osaka-u.ac.jp (K. Hanagaki). 
The LHC delivered first single beam in September 2008. The SCT succeeded in observing the first beam splash events produced by allowing a single pilot bunch to impact on collimators upstream of the experiment. The problems of the LHC following the accident on September 19th have meant that we have been waiting around a year for the restart of the LHC. We expect the LHC resuming operation in November 2009.

The LHC's design value of luminosity is $10^{34} \mathrm{~cm}^{2} \mathrm{~s}^{-1}$. The bunch spacing is $25 \mathrm{~ns}$. From these two parameters it is understood that the SCT is required to be radiation hard, and have low occupancy in spite of the short bunch spacing and hence short integrating and/or shaping time at the front-end. In this paper, we describe the design of SCT $[1,2]$, the current status and the operational experience, and the performance recorded to date.

\section{Design}

There are four barrel layers, and nine disks per each endcap. The barrel covers $|\eta|$ less than 1.1-1.4 depending on the location, and the endcap covers $|\eta|$ greater than 1.1-1.4. The barrel consists of 2,112 modules, and the endcap consists of 988 modules per endcap, a total of 4,088 modules.

Each barrel module consists of four approximately square $(6 \times 6 \mathrm{~cm})$ p-on-n single sided silicon sensors; two sensors on each side are electrically connected to give 768 strips of approximately $12 \mathrm{~cm}$ long, mounted on the thermal and mechanical support. The strip pitch is $80 \mu \mathrm{m}$. Another pair of sensors is glued back-to-back to the first pair again on the support with the stereo angle of $40 \mathrm{mrad}$, giving a two dimensional determination of hit position. The six ABCD3TA [3] readout chips are mounted on a multi-layer $\mathrm{Cu}$-polyimide flex circuit for each side. There are 12 readout chips or 1536 strips per module, and hence approximately $3.2 \mathrm{M}$ readout channels for the barrel.

The endcap module has similar structure to the barrel. The difference is a wedge shape of the sensor to build up the disk shape, hence there are different sizes of sensor or module depending on the location, i.e. whether it sits on inner, middle, or outer part of the disk. The strip pitch varies from 57 to $94 \mu \mathrm{m}$. Again there are 12 readout chips per module, resulting in approximately $1.5 \mathrm{M}$ readout channels for each endcap.

The grand total of the number of readout strips is $6.3 \mathrm{M}(4088$ modules $\times 12$ chips $\times 128$ channels per chip).

In order to minimize the size of the data read out when a charged particle crosses the tracking detectors, the ABCD3TA front end chip is designed to send only the channel address for the strips that are above a pre-defined (programmable) threshold.

The total heat load per module is $5.6 \mathrm{~W}$ before irradiation. To sustain the radiation damage up to $2 \times 10^{14}$ neutron equivalence per $\mathrm{cm}^{2}$ at the innermost layer, the sensors are cooled down to $-7^{\circ} \mathrm{C}$ by a two-phase cooling system using $\mathrm{C}_{3} \mathrm{~F}_{8}$ as a coolant. At the 
same time, the sensor can sustain up to $480 \mathrm{~V}$ bias voltage to be fully depleted after the total flux we expect to see over the 10 year lifetime of the SCT.

The communication between the modules and off-detector electronics is established by optical links. The Back Of Crate (BOC) card plays the role of the interface between the Read Out Driver (ROD) module and the optical links on the modules. For each module, clock and command signals are sent from the BOC by a single optical fibre, while the data from the module are sent back to BOC by a pair of fibres one corresponding to each side of the module. The clock and command signal is transmitted to the module by the TX plug-in, and the data on the module is received by the RX.

As the detector is not accessible once the whole ATLAS detector is integrated, a readout redundancy scheme is important. On the module, the six readout chips are daisy-chained, and read out by one optical fibre, as mentioned above. In case a chip is not functional, the readout chain can be configured to bypass the malfunctioning chip. In case of broken optical fibre for data readout, the data can be routed to an optical link for the other side on the module. Finally, there is a spare route for clock and command signals. In 2008 there were a series of failures of the off-detector TX transmitters due to ESD damage in construction. Extensive use was made of the redundancy scheme while waiting for the manufacture and installation of the replacement units. The units were replaced in July and August 2009.

\section{Status and Operational Experience}

The first beams were injected into the LHC on September 10th 2008. The beams were injected separately in both directions (clockwise and counter-clockwise) without ramping. At predefined times the single beam was allowed to hit the collimator and created a beam splash consisting of thousands of particles entering the ATLAS detector. The endcap observed many space hit points, although the bias voltage was set to $20 \mathrm{~V}$ which is lower than the nominal value of $150 \mathrm{~V}$ for reasons of safety. This indicates that the SCT was integrated to the ATLAS DAQ in proper timing, and was ready for beam in time.

Unfortunately the failure of the bus-bars connecting two superconducting magnets and the subsequent collateral damage and helium leak has stopped the LHC program for more than one year. Using the long LHC down time, the SCT collaboration continued to systematically check and improve all of its control systems. Table 1 shows the fraction of non-functional channels relative to the total 6.3M readout channels. As can be seen, more than $99 \%$ of the channels are active. There is one cooling loop which is not functional because of the leak inside the detector. The modules mounted on this loop are not powered on, although the modules themselves are functional. The two modules are not functional because there is an open circuit in bias in one module and short to ground on the control line in the other. The 10 chips cannot be read out, because a master chip in each link in the barrel cannot be read out with the redundancy scheme where one side of the optical 
fibre is broken, i.e. this is attributed to 10 bad optical links, not the problem of chips themselves. The remaining problem in strips is mainly high noise. However, the number of bad strips per module is mostly less than 15 which meets our design specification.

In terms of operational experience, there were two major issues. The first one was mentioned above and relates to the TX plug-in for the optical links. There seems to have been ESD damage in production and the SCT observed the number of non-functional TX plug-in gradually increase. As of writing of this paper, all the damaged TX plug-in have been replaced.

The second one was related to the cooling. The failure of three out of six compressors on May 1st 2008 was a severe set-back. The three compressors were repaired within three months and most of the pipe-work in the system was replaced or cleaned. Additional problems of excessive vibrations affecting all six compressors meant that all of the compressors were retrofitted by the vendor in the winter of 2008-2009.

The only remaining issue is related to the failure of some large area heaters pads between the SCT and the TRT. These pads are designed to maintain the thermal neutrality between the SCT and TRT. Without these pads the cold SCT would cool down the TRT below its design operational temperature. Therefore, the outer part of the SCT Barrel operates the coolant at $-10^{\circ} \mathrm{C}$ instead of $-25^{\circ} \mathrm{C}$. But this problem affects only the outer layer of the Barrel. We don' t expect a significant impact on the lifetime, because the innermost layer, which is expected to have the severest radiation damage, can still run cold.

\section{Performance}

Since no analogue information is read out, one of the most useful and important parameters that quantifies the detector performance is noise occupancy. At the same time, the detector calibration of parameters such as gain or threshold determination is performed by injecting test charges of various sizes and monitoring the change in occupancy. Assuming noise to have Gaussian shape, one can extract equivalent noise charge (ENC) by monitoring occupancies for different thresholds. The measured ENC is shown in Fig 2. There should be five groups in this distribution, because there are five different sizes of sensors used in the SCT, and hence the different capacitive load to the preamp. The short sensors at the middle endcap, the inner endcap, the middle endcap, and the outer endcap can be identified in the plot with the peak positions around 940,1100, 1580, and 1660 electrons, respectively. The barrel was not cooled down to the nominal operational temperature when these data were taken, and the inner and outer layers had the different temperature. This resulted in the two peaks. The ENC' s were 1510 for the inner barrel, and 1550 electrons for the outer barrel. This temperature dependence is in good agreement with the expectation or the design value.

The ENC discussed above is measured by the SCT calibration procedure. At the same time 
the real-time detector performance has to be monitored throughout a global physics run by the noise occupancy extracted from the data. Here the noise occupancy is calculated by subtracting the coincident hits from two sides of sensor in a module (the hits constituting a space point) from the total number of hits. Thus, the noise occupancy in calibration runs was compared and confirmed to be consistent with the one obtained in physics runs, implying the quoted ENC above can be a good representative of the noise performance. Also the number of noisy strips, defined by a strip with higher occupancy above a certain threshold, was monitored for long term. This study shows the noise level was stable for a year or so.

The intrinsic hit efficiency of the barrel module was measured using cosmic ray muons. The tracks were required to have $10 \mathrm{SCT}$ hits, 30 TRT hits and a $\chi^{2} / \mathrm{DOF}$ of track fitting smaller than two. Furthermore their intersection with the modules had to be within $40^{\circ}$ of normal incidence. Finally a guard region around the edge of the active silicon was excluded. The result is shown in Fig. 3 as a function of layer (each layer has two single sided sensors glued back-to-back, thus there are eight entries). The silicon efficiency was then found to be $99.75 \%$ in average.

Again using cosmic ray muons, the Lorentz angle in 2 Tesla magnetic field was measured in-situ. This was done by counting the average cluster size as a function of muon's incident angle to the module. The angle which gives the smallest cluster size was determined to be the Lorentz angle. It is measured to be $3.93^{\circ} \pm 0.03^{\circ}$ (stat.) $\pm 0.10^{\circ}$ (syst.) in data, while $3.69^{\circ} \pm 0.19^{\circ}$ (syst.) in Monte Carlo expectation. This result together with the previous results obtained in test beam with different magnetic field is shown in Fig. 4. The good agreement with the prediction in three different magnetic fields indicates the validity of the modeling of electron/hall mobility in the silicon sensor.

\section{Conclusions}

The SCT was installed into the ATLAS detector between 2006 and 2007. A huge effort has gone into the installation and commissioning of the detector by the SCT collaboration. As a result, more than $99 \%$ out of $6.3 \mathrm{M}$ readout channels are functional. The noise level has been carefully measured and monitored to confirm that it is consistent with the design expectations. The hit efficiency has been measured to be far above $99 \%$ in average. The only current issue is that the outer layer of the barrel needs to run in slightly higher temperature, but this should not affect the whole lifetime of the SCT. In conclusion, the SCT shows an excellent performance, and is ready for beam collision and physics data taking. 


\section{Acknowledgment}

I acknowledge the members of ATLAS SCT group.

\section{References}

[1] G. Aad et al. (ATLAS Collaboration), JINST 3, S08003 (2008).

[2] A. Abdesselam et al. (ATLAS SCT group), JINST 3, 10006 (2008).

[3] F.Campabadal et al., Nucl. Inst. and Meth. A 552, 292 (2005). 


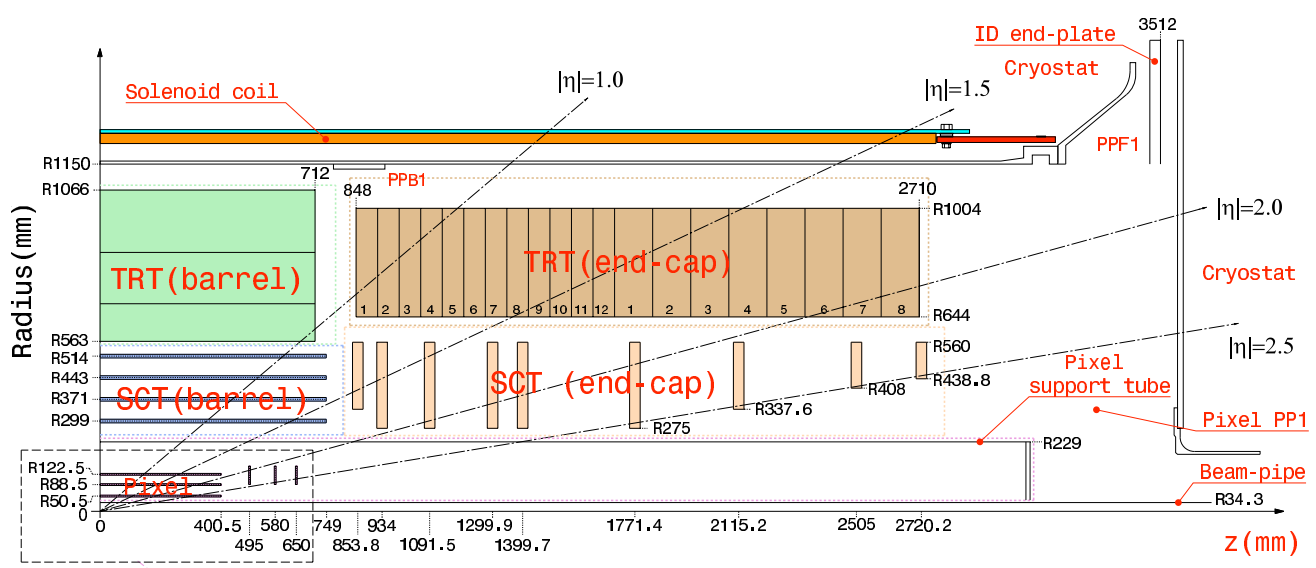

Fig. 1. The plane view of the charged particle tracking system at the ATLAS.

Table 1

Fraction of non-functional components.

\begin{tabular}{l|l|l} 
disabled components & Not expected to be resolved & Yet unresolved \\
\hline cooling loop & $0.32 \%$ (one loop) & 0 \\
module & $0.05 \%$ (two modules) & $0.42 \%(17$ modules $)$ \\
chip & $0.02 \%(10$ chips $)$ & $0.03 \%(16$ chips $)$ \\
strip & $0.16 \%(10,151$ strips $)$ & 0 \\
\hline total & $0.55 \%$ & $0.35 \%$
\end{tabular}

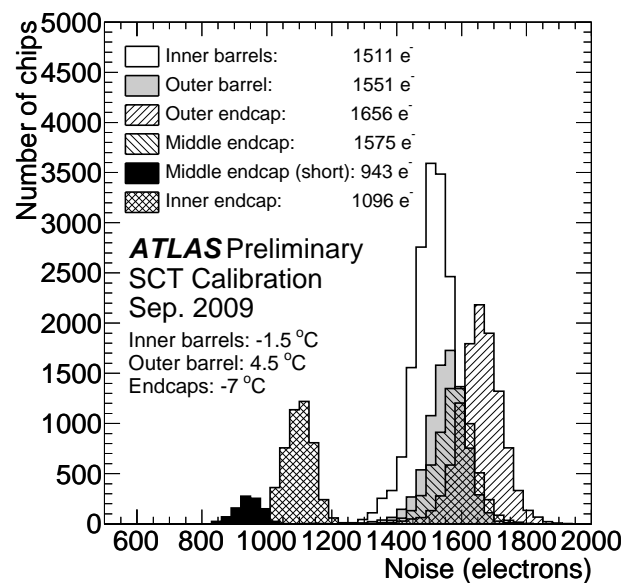

Fig. 2. The ENC measured for each readout chip. 


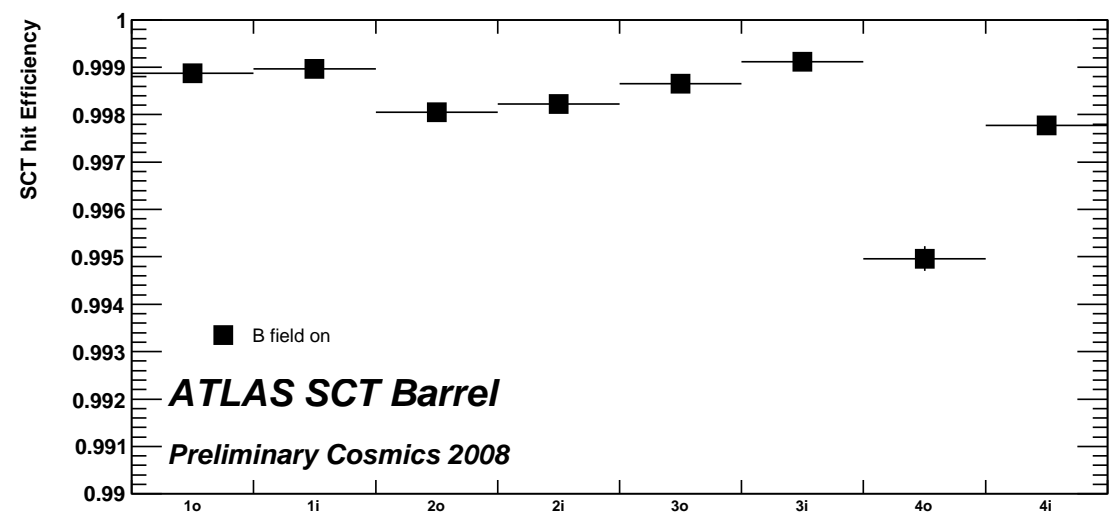

Fig. 3. The efficiency of the barrel measured by cosmic ray. Each two points correspond to a layer consisting of two sides of single sided sensor. The most left (right) two points show the efficiency of the innermost (outermost) layer.

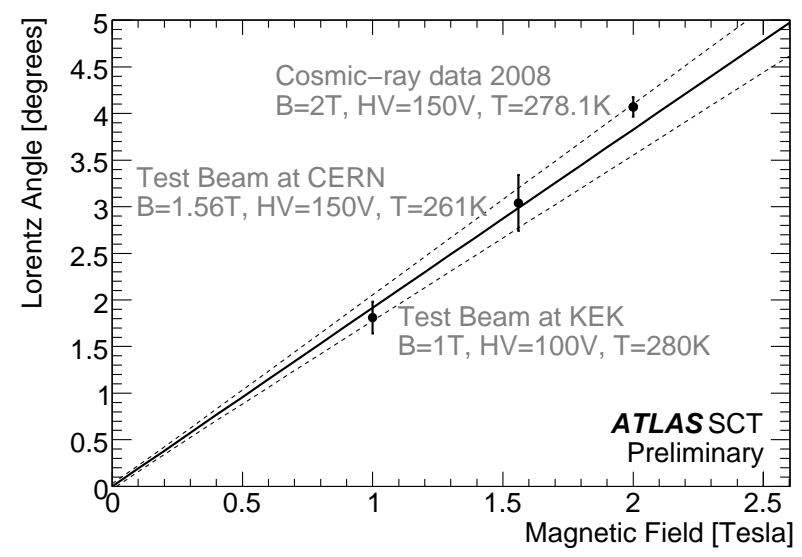

Fig. 4. The Lorentz angle as a function of magnetic field. The point on 2 Tesla is obtained in-situ from the cosmic ray data taken in 2008 . The other two points are test beam results. 